\title{
Smallest Universe of Negative Curvature
}

\author{
Helio V. Fagundes \\ Instituto de Física Teórica, Universidade Estadual Paulista, Rua Pamplona, 145, São Paulo, SP 01405-900, Brazil
}

(Received 4 December 1992)

\begin{abstract}
The smallest known three-dimensional closed manifold of curvature $k \equiv-1$ was discovered a few years ago by Weeks. This kind of manifold is constructed from a hyperbolic polyhedron with faces pairwise identified. Here it is used as the comoving spatial section of a Friedmann cosmological model, in the spirit of Ellis and Schreiber's idea of small universes. Its nontrivial global topology has the effect of producing multiple images of single cosmic sources, and this is the basis of an attempt to solve a famous controversy about the redshifts of quasars.
\end{abstract}

PACS numbers: $98.80 . \mathrm{Hw}, 04.20 . \mathrm{Jb}, 98.54 . \mathrm{Aj}, 98.62 . \mathrm{Py}$

The idea that cosmic space may have a nontrivial global topology, being closed (i.e., finite and boundless) for any sign of the spatial curvature, has had a slow but sustained development since Ellis's pioneer paper [1]. More recent literature can be traced from Fagundes [2]. The usual assumptions of theoretical cosmology, homogeneity and isotropy of matter distribution (cf. Landau and Lifshitz [3], for example), combined with general relativity, only determine the local spacetime metric, but not the global topology of spacetime. For subcritical densities $(\Omega<1)$ and null cosmological constant $\Lambda$, the Friedmann-Robertson-Walker metric is $d s^{2}=-c^{2} d t^{2}$ $+a^{2}(t) d \lambda^{2}$, where

$$
d \lambda^{2}=d \chi^{2}+\sinh ^{2} \chi\left(d \chi^{2}+\sin ^{2} \theta d \varphi^{2}\right)
$$

is the metric of comoving space, with constant curvature $k=-1$. The usual comoving spatial section attributed to this solution is the open, simply connected, infinite hyperbolic space $H^{3}$, but there is an infinity of closed, multiply connected hyperbolic manifolds [4] (CHMs) that can be taken as that spatial section. A CHM is usually constructed [1] from a fundamental polyhedron (FP) or Dirichlet region in $H^{3}$, with faces pairwise identified. The FP determines a tessellation or honeycombing [5] of $H^{3}$ into cells which are replicas of the FP, through the action of a discrete group of isometries related to the face pairings (see below) and to the manifold's topology.

Some time ago the author (Fagundes [6]; henceforth that paper will be referred to here as QGAII, after its title) tried to solve the quasar redshift controversy [7] through a model with nontrivial topology of three-space: The multiple images of single sources predicted by these universes might explain the presumed associations of quasars and galaxies with discordant redshifts. That model's CHM had a hyperbolic icosahedron as FP. The results were qualitatively interesting, but quite off the observed redshifts: In hyperbolic geometry one has no freedom to fix the scale of such polyhedra, because their dihedral angles are fixed by the requirements of the mentioned tessellation; and the big size of that icosahedron (volume $V=4.686$. . in the usual normalization of the curvature to $k=-1$ ) did not allow the production of Arp's pairs with realistic redshifts.

In this paper I build a similar model [8] with the smallest CHM that is known to geometers at present, $V$ $=0.9427 \ldots$ This manifold was discovered by Weeks [9], so it will be referred to as the Weeks manifold (WM). Its small size is particularly interesting to cosmology, in the light of Ellis and Schreiber's [10] proposal of a small universe and of the above remark about the redshifts of quasars associated with galaxies. My choice of a subcritical density, $\Omega=0.1$, is mainly dictated by the stated concern of finding a theory for these and similar associations as a topological effect, but also by a desire to explore techniques for dealing with the geometry of CHMs. Note that a low value for $\Omega$ is consistent with astrophysical observations and inferences, although theoreticians have been giving preference to the critical value $\Omega=1$, based on speculative forms of dark matter.

The FP for the Weeks manifold is an 18-sided figure whose 26 vertices' Minkowski coordinates in my representations are listed in Table I. These coordinates are related to the spherical ones in Eq. (1) by $X_{1}=\sinh \chi \sin \theta$ $\times \cos \phi, X_{2}=\sinh \chi \sin \theta \sin \phi, X_{3}=\sinh \chi \cos \theta, X_{4}=\cosh \chi$, point $\chi=0$ being the centroid of the FP. They are very useful for handling rigid motions in $H^{3}$, for this space is imbedded in an abstract Minkowski space as the upper branch of a hyperboloid [3]. So a rigid motion in $H^{3}$ is expressed as a proper, isochronous Lorentz transformation [11]. The group $\Gamma$ which produces the tessellation for WM is generated by 18 such transformations $\gamma_{k}$, $k=0-17$. Each of these is a motion that takes the FP to a replica $\gamma_{k}$ (FP), which has a face in common with the original; the faces are so numbered that for $k=0-8$, $\gamma_{k}\left(F_{k+9}\right)=\gamma_{k}(\mathrm{FP}) \cap \mathrm{FP}=F_{k}$ and $\gamma_{k+9}=\gamma_{k}^{-1}$. Table II lists the faces of FP, and the generators that link them, expressed in terms of two more basic motions [12], $a$ and $b$, given in Table III.

Ellis and Schreiber [10] mention several advantages of the cosmic three-space being closed and small. Among these is the fact that it helps explain the isotropy and homogeneity of cosmic observations, with or without the inflationary scenario. One may add that the production of multiple images, besides serving our current purpose 
TABLE I. The Minkowski coordinates for the 26 vertices of the fundamental polyhedron for the Weeks manifold, which is the comoving spatial section of this paper's model.

\begin{tabular}{lrrrr}
\hline \hline & \multicolumn{1}{c}{$X_{1}$} & \multicolumn{1}{c}{$X_{2}$} & \multicolumn{1}{c}{$X_{3}$} & \multicolumn{1}{c}{$X_{4}$} \\
\hline $\mathrm{G}$ & 0.00000000 & 0.00000000 & 0.81159257 & 1.28789848 \\
$\mathrm{O}$ & 0.00000000 & 0.10664201 & 0.78401962 & 1.27517029 \\
$\mathrm{~A}$ & 0.47654246 & 0.27513192 & 0.61537675 & 1.29671849 \\
$\mathrm{~B}$ & 0.62025991 & -0.16851487 & 0.16978597 & 1.20081093 \\
$\mathrm{D}$ & 0.43218382 & 0.62623778 & 0.21703147 & 1.27517029 \\
$\mathrm{~T}$ & 0.68871325 & 0.03742749 & -0.38772738 & 1.27517029 \\
$\mathrm{C}$ & 0.54663073 & 0.54283761 & -0.29664965 & 1.29671849 \\
$\mathrm{X}$ & 0.65134973 & -0.00044773 & -0.48417540 & 1.28789848 \\
$\mathrm{~W}$ & 0.57995255 & 0.06697606 & -0.53406790 & 1.27517029 \\
$\mathrm{R}$ & -0.09235469 & -0.05332100 & 0.78401962 & 1.27517029 \\
$\mathrm{H}$ & -0.47654246 & 0.27513192 & 0.61537675 & 1.29671849 \\
$\mathrm{~L}$ & -0.16419180 & 0.62141827 & 0.16978597 & 1.20081093 \\
$\mathrm{I}$ & -0.75842974 & 0.06116328 & 0.21703147 & 1.27517029 \\
$\mathrm{Q}$ & -0.37676978 & 0.57772942 & -0.38772738 & 1.27517029 \\
$\mathrm{~K}$ & -0.74342652 & 0.20197730 & -0.29664965 & 1.29671849 \\
$\mathrm{Y}$ & -0.32528712 & 0.56430928 & -0.48417540 & 1.28789848 \\
$\mathrm{U}$ & -0.34797925 & 0.46876561 & -0.53406790 & 1.27517029 \\
$\mathrm{~S}$ & 0.09235469 & -0.05332100 & 0.78401962 & 1.27517029 \\
$\mathrm{E}$ & 0.00000000 & -0.55026383 & 0.61537675 & 1.29671849 \\
$\mathrm{~J}$ & -0.45606811 & -0.45290341 & 0.16978597 & 1.20081093 \\
$\mathrm{~F}$ & 0.32624592 & -0.68740106 & 0.21703147 & 1.27517029 \\
$\mathrm{P}$ & -0.31194347 & -0.61515691 & -0.38772738 & 1.27517029 \\
$\mathrm{M}$ & 0.19679579 & -0.74481490 & -0.29664965 & 1.29671849 \\
$\mathrm{Z}$ & -0.32606261 & -0.56386155 & -0.48417540 & 1.28789848 \\
$\mathrm{~V}$ & -0.23197331 & -0.53574168 & -0.53406790 & 1.27517029 \\
$\mathrm{~N}$ & 0.00000000 & 0.00000000 & -0.66479086 & 1.20081093 \\
\hline \hline & & & &
\end{tabular}

about the redshift controversy, is quite interesting by itself, for it would give us information about different epochs of the sources' evolution. And that, according to quantum cosmologists, the probability for spontaneous creation of a small universe is bigger than for a large one, being null for infinite universes [13].

Figure 1 is similar to but more detailed than Fig. 3 in Ref. [10], in that it represents the simulated distribution [14] of images produced by 31 sources in WM as cosmic space. In these models it is the distribution of images, not sources, that is expected to be approximately homogeneous and isotropic [15]. One of the sources is the Milky Way galaxy at point $P_{0}$ (the Earth's, or observer's,
TABLE II. The faces of the fundamental polyhedron and the generators that relate them: $F_{k}=\gamma_{k}\left(F_{k+9}\right), k=0-8$. $a$ and $b$ are defined in Table III.

\begin{tabular}{llll}
\hline \hline$k$ & $F_{k}$ & $F_{k+9}$ & \multicolumn{1}{c}{$\gamma_{k}$} \\
\hline 0 & ABTCD & HLDAO & $a^{2} b^{-1}$ \\
1 & SEFBA & FMPJE & $a b$ \\
2 & RHIJE & IKQLH & $b^{-1} a b^{-1}$ \\
3 & FMXTB & UKZVN & $a$ \\
4 & WNUYC & IJPZK & $b$ \\
5 & CYQLD & MXWNV & $b a^{-1}$ \\
6 & CTXW & HOGR & $b^{2} a^{-1} b$ \\
7 & KQYU & ERGS & $b a^{-1} b^{2} a^{-1} b$ \\
8 & MPZV & ASGO & $b^{-1} a^{-1} b^{-1} a-1$ \\
\hline \hline
\end{tabular}

position), the others being at points $P_{n}, n=1-30$, chosen pseudorandomly. With each generator applied to these points we get $\gamma_{k}\left(P_{n}\right), k=0-17, n=0-30$, as potential [16] indirect images; the figure represents all images with redshift $Z \leq 3.1$, as seen from $P_{0}$. The apparent anisotropy in the figure with some voids and concentrations is suggestive but is not meaningful in the present context, because we only have a partial covering of the space of images $H^{3}$, namely the FP itself and the 18 cells $\gamma_{k}$ (FP). Now, a degree of anisotropy is expected in such cosmologies, as explained in QGAII and in agreement with Arp's observations [17]. Kokubun and I [18] are working on a more detailed computer simulation of the QGAII model, to estimate this anisotropy in the case of quasar-galaxy associations. In a future study a complete covering of the observable cosmos will be made using the present theory, to try to explain topologically the small anisotropy detected by NASA's COBE satellite in the $2.7 \mathrm{~K}$ background radiation [19].

As in QGAII, to interpret Arp's associations as conjunctions of pairs of images of single sources, we need to determine self-crossing geodesics in the comoving space. To find these, one scans each face of the FP, looking for points that, linked to Earth's position, produce selfcrossing geodesics in the FP. The method is given in detail in Appendix B of QGAII, except that here we first triangulate the faces, which are either pentagonal or quadrilateral-cf. Table II. Earth's position was taken to be $\chi=0.4, \theta=15^{\circ}, \varphi=30^{\circ}$. As in Fig. 1, solutions with

TABLE III. The basic matrices in terms of which generators $\gamma_{k}$ are expressed in Table II.

\begin{tabular}{|c|c|c|c|c|}
\hline \multirow{4}{*}{$a=$} & 1.08002794 & -0.23611344 & 0.89642406 & 1.01281091 \\
\hline & 0.01223938 & -0.72016273 & -0.96925233 & -0.67693001 \\
\hline & 0.47017146 & 0.65634321 & -0.62440977 & -0.20429190 \\
\hline & 0.62263260 & 0.07190498 & 1.06438418 & 1.58926252 \\
\hline \multirow{4}{*}{$b=$} & -0.17317475 & 0.95965035 & 0.33332416 & 0.24904476 \\
\hline & 0.71129753 & 0.53303996 & -0.73535204 & 0.57516814 \\
\hline & -1.28760915 & -0.29169981 & -0.62440977 & -1.06438418 \\
\hline & 1.09264404 & 0.53865497 & 0.20429190 & 1.58926252 \\
\hline
\end{tabular}




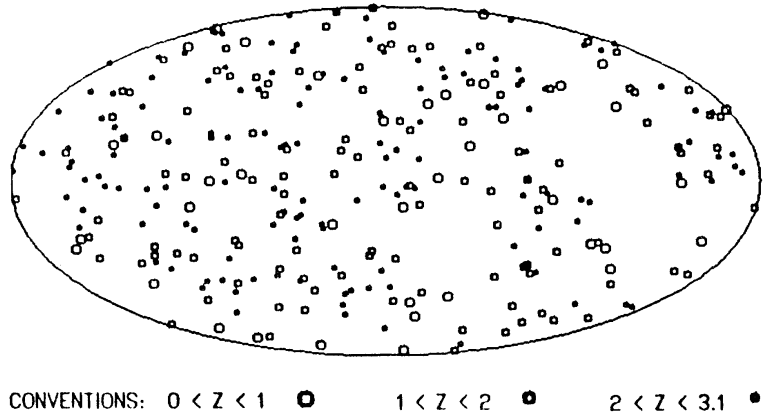

FIG. 1. Celestial map of images produced by our Galaxy and thirty other "galaxies," in a simulation according to the model. Earth's position in the fundamental polyhedron is $\chi=0.40$, $\theta=15^{\circ}, \varphi=30^{\circ}$

$Z>3.1$ were excluded.

The results [20] are presented in Table IV as exact conjunctions of images, but of course observed separations of up to a few arcminutes should be regarded as good data for comparison. Note that the rays producing these second images travel long paths of the same length as the distances of the corresponding images, and are sometimes scattered, sometimes focused by intervening gravitational fields. Each scanned face or search region produces a continuous line segment, whose points are the potential positions of objects in conjunction with one of its topologically generated images; the positions in the table are sample points along these segments-cf. Fig. 5 in QGAII.

A fit to the quasar-galaxy pairs in Burbidge et al. [21], for example, would be quite unreliable at this point, since besides arbitrary parameters in the model (Earth's position in the FP, the orientation of the axes, and the values of $\Lambda$ and $\Omega$ ), only a fraction of the observed pairs can be attributed to a topological effect, the majority being lineof-sight coincidences. One would like to see a deeper study of the observed pairs that might reveal evolutionary traits in a number of them. Then we would get a separation of accidental versus topological associations, and a meaningful fit of the model could become feasible.

However, the redshifts listed for the associations in Table IV are quite realistic, and the fact that the conjunctions occur in aligned groups also reflects some of Arp's claims, at least qualitatively. For example, the images produced by search region 10 have redshifts in ranges similar to those of the grouping of galaxies, quasars, and radio sources near galaxy M33. It is true that the group of region 10 covers a linear range of about $22^{\circ}$, with two classes of smoothly varying redshifts; while in Arp [7] the group is quite scattered about a line, and has a range of $50^{\circ}$. On the other hand, there are tight groups, like the three quasistellar objects $(Z=2.048$, $2.054,2.040)$ about the irregular galaxy $\operatorname{M} 82(Z$ $=0.001$ ) [22], for which I gave a tentative interpretation
TABLE IV. Sample values of potential positions for two images of single sources in conjunction: $Z_{1}, Z_{2}$ are their redshifts; lon, lat their theoretical longitude and latitude.

\begin{tabular}{|c|c|c|c|c|}
\hline $\begin{array}{l}\text { Search } \\
\text { region }\end{array}$ & $Z_{1}$ & $Z_{2}$ & lon & lat \\
\hline \multirow[t]{7}{*}{0} & 0.800 & 2.591 & 15.56 & -42.82 \\
\hline & 0.516 & 2.041 & 19.28 & -41.18 \\
\hline & 0.337 & 1.723 & 22.99 & -39.11 \\
\hline & 0.215 & 1.531 & 26.42 & -36.67 \\
\hline & 0.123 & 1.409 & 29.51 & -33.86 \\
\hline & 0.046 & 1.335 & 32.22 & -30.67 \\
\hline & 0.002 & 1.306 & 33.75 & -28.36 \\
\hline \multirow[t]{5}{*}{1} & 0.001 & 1.509 & 346.24 & 28.45 \\
\hline & 0.048 & 1.519 & 347.53 & 30.77 \\
\hline & 0.119 & 1.560 & 349.72 & 33.84 \\
\hline & 0.205 & 1.643 & 352.46 & 36.81 \\
\hline & 0.308 & 1.777 & 355.50 & 39.41 \\
\hline \multirow[t]{5}{*}{2} & 0.008 & 2.065 & 230.27 & 12.20 \\
\hline & 0.094 & 2.017 & 231.24 & 15.72 \\
\hline & 0.231 & 2.039 & 233.34 & 20.69 \\
\hline & 0.373 & 2.158 & 235.75 & 24.75 \\
\hline & 0.536 & 2.370 & 238.28 & 28.10 \\
\hline \multirow[t]{6}{*}{9} & 0.002 & 1.306 & 127.02 & 19.21 \\
\hline & 0.021 & 1.317 & 127.41 & 20.33 \\
\hline & 0.095 & 1.379 & 129.06 & 24.09 \\
\hline & 0.189 & 1.494 & 131.24 & 27.76 \\
\hline & 0.246 & 1.577 & 132.48 & 29.46 \\
\hline & 0.356 & 1.755 & 134.56 & 31.92 \\
\hline \multirow[t]{7}{*}{10} & 1.001 & 3.046 & 251.20 & -35.66 \\
\hline & 0.761 & 2.569 & 252.81 & -34.09 \\
\hline & 0.526 & 2.131 & 255.14 & -31.50 \\
\hline & 0.393 & 1.906 & 256.91 & -29.18 \\
\hline & 0.239 & 1.683 & 259.41 & -25.17 \\
\hline & 0.050 & 1.520 & 262.59 & -17.29 \\
\hline & 0.000 & 1.509 & 263.22 & -14.57 \\
\hline \multirow[t]{8}{*}{11} & 0.949 & 3.085 & 149.40 & -36.25 \\
\hline & 0.718 & 2.664 & 148.91 & -34.29 \\
\hline & 0.482 & 2.294 & 152.42 & -30.97 \\
\hline & 0.199 & 2.025 & 157.80 & -23.75 \\
\hline & 0.021 & 2.054 & 160.69 & -17.01 \\
\hline & 0.002 & 2.070 & 160.91 & -16.23 \\
\hline & 0.001 & 2.071 & 160.92 & -16.19 \\
\hline & 0.001 & 2.071 & 160.93 & -16.18 \\
\hline
\end{tabular}

in QGAII in terms of a collision of quasar-centered galaxies to form young M82 (now invisible); if we look at search region 11 in Table IV we do see some numbers like these. The separation between M33 and M82 is not reproduced in this way, but the tendency remains for topologically nontrivial cosmologies to account for these and other clusterings with discordant redshifts.

Some of the unorthodox claims this theory did not confirm: material bridges or jets linking objects with discordant redshifts [7] - but see Fagundes [23]; groups with small, discordant redshifts, like Stephan's quintet of gal- 
axies, presumed to be physically associated-but Hickson, Kindl, and Huchra [24] have argued that there is a realistic chance that this could be just a coincidence of projections on the sky. Hayward and Twamley [25] use a lower bound for the volume of $\mathrm{CHMs}, V \geq 0.000082$; and, assuming a space really may exist with this minimal volume, they give a qualitative explanation of the periodicity observed by Broadhurst et al. [26] in the distribution of galaxies. The present model is not small enough to account for that and similar periodicities [27], so we would like to see the discovery of CHMs much smaller than Weeks'.

I am very grateful to Jeff Weeks for an extensive correspondence on CHMs and for his stimulating comments about their application to cosmology.

[1] G. R. R. Ellis, Gen. Relativ. Gravitation 2, 7 (1971).

[2] H. V. Fagundes, Phys. Rev. Lett. 54, 1200 (1985); Gen. Relativ. Gravitation 24, 199 (1992).

[3] L. Landau and E. M. Lifshitz, Classical Theory of Fields (Pergamon, Oxford, 1975), 4th ed., Chap. 14.

[4] W. P. Thurston, Bull. Am. Math. Soc. (New Series) 6, 357 (1982); W. P. Thurston and J. R. Weeks, Sci. Am 251, No. 1, 94 (1984).

[5] See H. S. M. Coxeter, Twelve Geometric Essays (Southern Illinois Univ. Press, Carbondale, IL, 1968), Chap. 10.

[6] H. V. Fagundes, Astrophys. J. 338, 618 (1989); 349, 678(E) (1990).

[7] H. Arp, Quasars, Redshifts and Controversies (Interstellar, Berkeley, CA, 1989).

[8] A brief summary of these results was communicated at the Thirteenth National Meeting on the Physics of Particles and Fields, held in Caxambu, MG, Brazil, 16-20 September 1992 (unpublished).

[9] J. R. Weeks. Ph.D. thesis, Princeton University, 1985.

[10] G. F. R. Ellis and G. Schreiber, Phys. Lett. A 115, 97 (1986).

[11] See N. V. Efimov, Higher Geometry (Mir, Moscow, 1980), Sec. 202; or Fagundes (Ref. [6]), Appendix A.

[12] Matrices $a$ and $b$ were adapted from Weeks' formalism (private communication). They are generators of a simpler presentation of $\Gamma$, and satisfy the relations $a b^{-1} a b a b a b^{-1} a=b a^{-1} b^{2} a^{-1} b a b a=1$. The $\gamma_{k}$ 's in the text satisfy similar relations, which are omitted here for brevity.

[13] See D. Atkatz and H. Pagels, Phys. Rev. D 25, 2065 (1982); Y. P. Goncharov and A. A. Bytsenko, Astrophys. 27, 422 (1989), and references therein.

[14] The map is an approximate equal area projection, based on axes $X_{1}^{\prime}$ parallel to the $X_{1}$ axes, with origin on Earth's position. Longitude increases from left to right, contrary to astronomical usage; but this is immaterial here, since the orientation of WM is arbitrary.

[15] H. V. Fagundes, Phys. Rev. Lett. 51, 517 (1983); Gen. Relativ. Gravitation 24, 199 (1992).

[16] They are potential in the sense described in Refs. [15]

[17] H. Arp, J. Astrophys. Astron. (India) 5, 31 (1983).

[18] H. V. Fagundes and F. Kokubun (to be published).

[19] See, for example, C. S. Powell, Sci. Am. 267, No. 1, 9 (1992).

[20] Calculations were performed on an HP 750 Apollo workstation, with double precision (16-digit) arithmetic. But the shorter figures in Tables I and III allow approximate checking of Tables II and IV.

[21] G. Burbidge, A. Hewitt, J. V. Narlikar, and P. Das Gupta, Astrophys. J. (Suppl. Series) 74, 675 (1990).

[22] E. M. Burbidge, V. T. Junkkarinen, A. T. Koski, H. E. Smith, and A. A. Hoag, Astrophys. J. (Lett.) 242, L55 (1980).

[23] H. V. Fagundes, Rev. Mexicana Astrohn. Astrof. 21, 79 (1990).

[24] P. Hickson, E. Kindl, and J. P. Huchra, Astrophys. J. (Lett.) 329, L65, 1988.

[25] G. Hayward and J. Twamley, Phys. Lett. A 149, 84 (1990).

[26] T. J. Broadhurst, R. S. Ellis, D. C. Koo, and A. S. Szalay, Nature (London) 343, 726 (1990).

[27] See, for example, G. Burbidge, in Proceedings of the Tenth Texas Symposium on Relativistic Astrophysics, edited by R. Ramaty and F. C. Jones [Ann. N.Y. Acad. Sci. 375, 123 (1981)]; L.-Z. Fang and H. Sato, Commun. Theor. Phys. (China) 2, 1055 (1983); Quasars, Redshifts and Controversies (Ref. [7]), Chap. 7. 